\title{
Mesoscopic theory for systems with competing interactions near a confining wall
}

\author{
A. Ciach \\ Institute of Physical Chemistry, Polish Academy of Sciences, 01-224 Warszawa, Poland
}

(Dated: January 3, 2020)

\begin{abstract}
Mesoscopic theory for self-assembling systems near a planar confining surface is developed. EulerLagrange (EL) equations and the boundary conditions (BC) for the local volume fraction and the correlation function are derived from the DFT expression for the grand thermodynamic potential. Various levels of approximation can be considered for the obtained equations. The lowest-order nontrivial approximation (GM) resembles the Landau-Brazovskii type theory for a semiinfinite system. Unlike in the original phenomenological theory, however, all coefficients in our equations and $\mathrm{BC}$ are expressed in terms of the interaction potential and the thermodynamic state. Analytical solutions of the linearized equations in GM are presented and discussed on a general level and for a particular example of the double-Yukawa potential. We show exponentially damped oscillations of the volume fraction and the correlation function in the direction perpendicular to the confining surface. The correlations show oscillatory decay in directions parallel to this surface too, with the decay length increasing significantly when the system boundary is approached. The framework of our theory allows for a systematic improvement of the accuracy of the results.
\end{abstract}




\section{INTRODUCTION}

Competing interactions may lead to a self-assembly into different aggregates that at sufficiently low temperature $T$ form periodic patterns on a mesoscopic length scale $[1-15]$. The sequence of the ordered phases at low $T$ is the same in block copolymer systems [16] and in systems containing particles interacting with the effective SALR potential that is attractive at short- and repulsive at large separations [4, 8-10]. This universal behavior follows from the fact that on a qualitative level both systems can be described by the Landau-Brazovskii functional (LB) [4, 6]. A typical example of the SALR potential is the effective interaction between charged spherical particles (colloidal particles, nanoparticles or globular proteins) in a solvent inducing strong short-range effective attraction between them [3, 17-20]. One should mention, however that in the SALR systems the ordered phases have not been observed experimentally yet [19]. On the other hand, the cubic phases that are only metastable in the LB theory, turned out to be stable in multicomponent mixtures containing surfactant or lipids [21 25]. Thanks to this universality, one can be guided by the properties of one system in studies of the properties of another system with inhomogeneities on a well-defined length scale, even though some deviations from the universal properties of self-assembling systems may exist. Here we focus on the SALR model that is particularly simple, because the solvent is treated as a structureless medium, and only one kind of particles with isotropic interactions need to be considered.

Because of the periodic structure on the mesoscopic length scale (a few or a few tens of particle diameter), confinement by solid surfaces or by interfaces may lead to significant structural changes. The structural transformations depend on the properties of the system boundaries, and on the compatibility between the symmetry and period of the ordered structure and the shape and size of the confinement. The effect of confinement on the structure, mechanical and thermal properties of the ordered phases in amphiphilic and SALR systems was studied by theory, simulations and experiment [23, 25 41]]. In particular, structures absent in the bulk can be induced by appropriate boundaries [35, 36, 42].

Less attention was paid to effects of a single planar wall on the disordered phase in the soft-matter systems [43 46]. In the disordered phase the density is position-independent, and the correlation function exhibits either a monotonic or an oscillatory decay, just as in the case of simple fluids, but the wavelength of the oscillatory decay is set by the ranges 
of the attractive and repulsive parts of the interactions in the SALR systems, not by the size of the particles. The snapshots, however, show that the structure of the disordered phase in simple fluids and in the SALR systems can be completely different. At low volume fractions $\zeta$ and/or high $T$, the particles are more or less homogeneously distributed in both cases. When a so called critical cluster concentration line in the $(\zeta, T)$ phase diagram is crossed in the SALR system, however, clusters with a well-defined size appear [30, 47]. The isolated particles ('monomers') still dominate until another structural crossover at a higher $\zeta$ is reached. At this crossover, the probability of finding a monomer is equal to the probability of finding a particle belonging to a cluster of the optimal size, and the specific heat takes a maximum [44]. Further increase of the volume fraction leads to another structural crossover to a percolating network of particles [11]. The nontrivial structure of the disordered phase, in particular the strong inhomogeneities on a well-defined length scale, suggest nontrivial effects of an attractive or repulsive surface on the disordered phase in the SALR system. Indeed, simulations show: (i) formation of inhomogeneous layer of particles adsorbed at the surface, followed by strong depletion of particles in the subsequent layer (ii) anomalous decrease of adsorption for increasing chemical potential (iii) much larger correlation length near the confining wall than in the bulk. All these anomalies occur when clusters dominate over the monomers [44, 45].

Due to the broken translational and rotational symmetries, the average volume fraction depends on the distance from the wall, $z$, and the correlation function between the points $\mathbf{r}_{1}$ and $\mathbf{r}_{2}$ depends on $z_{1}, z_{2}$ and the distance $r_{\|}$between the projections of the two points on the surface $z=0$. In addition, formation of a structure with periodic order in the lateral direction in the near-surface region cannot be ruled out [46]. Thus, the problem is very complex. Because of this complexity, there is a need for an approximate theory that could give at least qualitative predictions with a reasonable effort. The LB theory developed for the unconfined SALR system in Ref. [4, 6, 7, 48] correctly predicts the sequence of phases. The results can be obtained much more easily than in the standard DFT or liquid theories. Importantly, in the original phenomenological LB theory there is a number of free phenomenological parameters, but in the theory developed in Ref. [4, 6], all parameters are expressed in terms of the interaction potential, $\zeta$ and $T$. In addition, the high- $T$ part of the phase diagram obtained in MF theories is qualitatively different from the simulation results, whereas in this theory it agrees with simulations on a semi-quantitative level when 
the fluctuation contribution is added to the grand potential [48].

In this work we generalize the LB theory developed in Ref. [4, 6] to the case of a semiinfinite system. Broken translational and rotational invariance, however, makes the derivation of the theory more difficult. The theory is developed in sec III. We limit ourselves to the MF approximation. In MF, the internal energy of a disordered phase in bulk is approximated by $U=\frac{1}{2} \rho^{2} \int V(r) d \mathbf{r}$, where $\rho$ is the average density and $V(r)$ is the interaction potential. This kind of MF approximation is typically made in standard DFT theories. The above expression for $U$ gives the same internal energy for homogeneous and inhomogeneous systems with the same average density. However, when clusters of a size determined by the range of attraction, separated by a distance determined by the range of repulsion are formed, the energy is much lower. This is because much more pairs of particles corresponding to a minimum of $V(r)$, and much less pairs of particles corresponding to the maximum of $V(r)$ are present than in the case of homogeneously distributed particles. As a result, the internal energy obtained by averaging the energy of microstates with the proper probability distribution differs from the energy calculated for the average density. This lower internal energy is associated with mesoscopic fluctuations of density or volume fraction in the disordered phase, where the clusters are not localized and move almost freely. Let us imagine a fixed mesoscopic part of a system containing clusters or other aggregates. The mesoscopic size means in this context the size comparable with the length scale of inhomogeneities (size of the clusters). The local volume fraction (or the number of particles in the mesoscopic window) is significantly larger or smaller than the average volume fraction when a cluster enters or leaves the window, respectively. In the case of a homogeneous system, the mesoscopic fluctuations are much smaller. What distinguishes the homogeneous and inhomogeneous systems with the same volume fraction, is the variance of the local volume fraction, associated with larger density inside the clusters than between them. The MF theories, including the standard DFT, correctly predict the low- and the high-temperature properties, where either the periodic phases are formed, or the clusters are not yet well developed, and fluctuations of the local volume fraction described above do not play a primary role. The fluctuation contribution to the grand potential will be considered in a forthcoming article by combining the DFT and the statistical field theories, as in Ref. [48].

We start in sec $\llbracket$ from the standard DFT expression for the grand potential and transform it to an equivalent form that is more suitable for making approximations. In secs IIB 
and $\amalg \mathrm{IC}$ we derive Euler-Lagrange (EL) equations and the boundary conditions (BC) from our expression for the grand potential. In sec ID we derive equations for the average volume fraction, and for the periodic modulations of $\zeta$ in the planes parallel to the wall. The latter is not considered in the following sections. We focus on the short-range order as described by the correlation function. In sec $\llbracket \mathrm{IE}$ we present and discuss linearized EL equations. Finally, in sec $\llbracket \mathrm{IF}$ the EL for the correlation function is developed, and various approximate versions are discussed. The solutions of the obtained EL equations are in principle equivalent to the results of minimization of the functional that was a starting point of our derivation. However, these equations can be greatly simplified by following the steps leading to the LB

theory in the bulk [4, 6, 7]. In sec III such a generic model is developed for a semiinfinite system. The EL equation for the volume fraction (sec IIIA) and the correlation function (sec $\underline{\text { IIIB }}$ ) as well as the BC take a particularly simple form. The linearized equations can be easily solved analytically, and we discuss properties of these solutions on a general level. In sec IV our theory is applied to a double-Yukawa interaction potential. The shapes of the excess volume fraction and the correlation function are presented and discussed. We summarize in sec:V.

\section{DEVELOPMENT OF THE THEORY}

In this work we consider the effect of the wall on the local structure when the disordered phase is stable in the bulk, and far away from the confining surface, the volume fraction $\zeta_{b}$ of the particles is position-independent. We assume that the confining $(x, y)$ plane is at $z=0$, and the particles $z$-th coordinate is $z \geq 0$. We develop a mean-field (MF) theory and assume that the grand potential can be written in the form

$$
\beta \Omega=\beta U+\beta U_{\text {ext }}+\int d \mathbf{r}_{\|} \int_{0}^{\infty} d z \beta f_{h}\left(\zeta\left(\mathbf{r}_{\|}, z\right)\right)-\beta \mu \int d \mathbf{r}_{\|} \int_{0}^{\infty} d z \zeta\left(\mathbf{r}_{\|}, z\right)
$$

where $\mu$ is the chemical potential, $\beta=1 /\left(k_{B} T\right)$, and $k_{B}$ is the Boltzmann factor. The third term in Eq.(11) is the entropic contribution. In a popular approach, $f_{h}$ is the hard-core reference-system free energy in the local-density approximation. The first two terms in (1) are the contributions to the internal energy associated with interparticle interactions and an external potential, respectively, and are given by

$$
U[\zeta]=\int d \mathbf{r}_{\|} \int_{0}^{\infty} d z \int d \Delta \mathbf{r}_{\|} \int_{0}^{\infty} d \Delta z \zeta\left(\mathbf{r}_{\|}, z\right) V\left(\Delta r_{\|}, \Delta z\right) \zeta\left(\mathbf{r}_{\|}+\Delta \mathbf{r}_{\|}, z+\Delta z\right)
$$


and

$$
U_{e x t}=\int d \mathbf{r}_{\|} \int_{0}^{\infty} d z V_{e x t}\left(\mathbf{r}_{\|}, z\right) \zeta\left(\mathbf{r}_{\|}, z\right)
$$

In (2) and (3), $V$ and $V_{\text {ext }}$ denote the interparticle interactions, and the interactions between the particles and the wall, respectively. In the case of the structureless wall to which we will restrict our attention later, $V_{\text {ext }}$ depends only on $z$. We assume that the interparticle inter-

actions are spherically symmetric, and depend only on $r=\sqrt{r_{\|}^{2}+z^{2}}$, but for convenience we will consider the directions parallel and perpendicular to the confining surface separately.

We introduce the excess grand potential

$$
\Delta \beta \Omega[\zeta]=\beta \Omega[\zeta]-\beta \Omega_{b}\left[\zeta_{b}\right]
$$

where $\Omega_{b}\left[\zeta_{b}\right]$ is the grand potential of the considered system with the same volume in the bulk. The volume fraction in the bulk, $\zeta_{b}$, satisfies the equation

$$
\zeta_{b} \int d \Delta \mathbf{r}_{\|} \int_{-\infty}^{\infty} d \Delta z \beta V\left(\Delta r_{\|}, \Delta z\right)+\beta f_{h}^{\prime}=\beta \mu
$$

that follows from the minimization of the grand potential of the unconfined system with the position-independent volume fraction. Here and below, $f^{\prime}(\zeta)=d f / d \zeta$ is used for any function $f$. In the next subsection we transform $\Delta \beta \Omega[\zeta]$ to a form more convenient for approximations and analytical solutions.

\section{A. Derivation of a new version of the density functional}

The local volume fraction can be split into the bulk and the excess terms,

$$
\zeta\left(\mathbf{r}_{\|}, z\right)=\zeta_{b}+\Delta \zeta\left(\mathbf{r}_{\|}, z\right)
$$

where $\mathbf{r}_{\|}$is a two-dimensional vector in a plane parallel to the confining wall. In general, we do not exclude the possibility of the wall-induced long-range order in the directions parallel to the wall. By the long-range order in the directions parallel to the wall we mean a periodic structure in the $(x, y)$ plane. If the surface induces only short-range ordering reflected in the oscillatory decay of the correlation function in the $(x, y)$ plane, but not on the level of the one-point volume fraction or density, then $\Delta \zeta\left(\mathbf{r}_{\|}, z\right)$ is independent of $\mathbf{r}_{\|}$. In the directions parallel to the surface, either translational invariance or periodic structure can be expected, 
and it is convenient to consider the volume fraction and the interaction potential in the mixed Fourier- and real-space representation,

$$
\begin{gathered}
\tilde{\zeta}\left(\mathbf{k}_{\|}, z\right)=(2 \pi)^{2} \delta\left(\mathbf{k}_{\|}\right) \zeta_{b}+\Delta \tilde{\zeta}\left(\mathbf{k}_{\|}, z\right) \\
\Delta \tilde{\zeta}\left(\mathbf{k}_{\|}, z\right)=\int d \mathbf{r}_{\|} e^{i \mathbf{k}_{\|} \cdot \mathbf{r}_{\|}} \Delta \zeta\left(\mathbf{r}_{\|}, z\right)
\end{gathered}
$$

and

$$
\tilde{V}\left(\mathbf{k}_{\|}, \Delta z\right)=\int d \mathbf{r}_{\|} e^{i \mathbf{k}_{\|} \cdot \mathbf{r}_{\|}} V\left(\mathbf{r}_{\|}, \Delta z\right)
$$

Here and below, tilde denotes a two-dimensional Fourier transform in the plane parallel to the confining surface, and $\delta$ denotes the Dirac delta function.

In the mixed representation, the excess grand potential is given by

$$
\begin{aligned}
\Delta \beta \Omega[\zeta] & =\int \frac{d \mathbf{k}_{\|}}{(2 \pi)^{2}} \int_{0}^{\infty} d z \int_{-\infty}^{\infty} d \Delta z \Delta \tilde{\zeta}\left(-\mathbf{k}_{\|}, z\right) \beta \tilde{V}\left(\mathbf{k}_{\|}, \Delta z\right) \theta(\Delta z) \Delta \tilde{\zeta}\left(\mathbf{k}_{\|}, z+\Delta z\right) \\
& +\int d \mathbf{r}_{\|} \int_{0}^{\infty} d z g\left(\zeta_{b}, \Delta \zeta\right)+\beta U_{\text {ext }}
\end{aligned}
$$

where $\theta(\Delta z)$ is the Heaviside unit step function,

$$
\begin{gathered}
g\left(\zeta_{b}, \Delta \zeta\right)=\beta f_{h}\left(\zeta_{b}+\Delta \zeta\right)-\beta f_{h}\left(\zeta_{b}\right)-\beta f_{h}^{\prime}\left(\zeta_{b}\right) \Delta \zeta \\
U_{e x t}=\int d \mathbf{r}_{\|} \int_{0}^{\infty} d z V_{e x t}\left(\mathbf{r}_{\|}, z\right) \zeta\left(\mathbf{r}_{\|}, z\right)=\int \frac{d \mathbf{k}_{\|}}{(2 \pi)^{2}} \int_{0}^{\infty} d z \tilde{V}_{e x t}\left(-\mathbf{k}_{\|}, z\right) \tilde{\zeta}\left(\mathbf{k}_{\|}, z\right),
\end{gathered}
$$

and Eq.(5) was used. In the case of a homogeneous confining surface, with no lateral pattern, the wall-particle interactions are independent of $r_{\|}, V_{\text {ext }}\left(\mathbf{r}_{\|}, z\right)=V_{\text {ext }}(z)$, and we get from (12) $U_{\text {ext }}=\int_{0}^{\infty} d z V_{\text {ext }}(z) \tilde{\zeta}(\mathbf{0}, z)$. If, in addition the external potential is of very short range, $U_{\text {ext }}$ can be approximated by

$$
U_{\text {surf }}=h \tilde{\zeta}(\mathbf{0}, 0)
$$

In the rest of this section we will transform the functional (10) to a form that is suitable for making approximations based on physical properties of the system. Our procedure is similar to the one developed in Ref. [4, 6] for the bulk inhomogeneous system.

In the first step we consider the internal energy contribution in Eq.(10). The Fourier transform of $\tilde{V}_{s}\left(\mathbf{k}_{\|}, \Delta z\right)=\tilde{V}\left(\mathbf{k}_{\|}, \Delta z\right) \theta(\Delta z)$ in the perpendicular direction contains both the real and the imaginary part

$$
\hat{V}_{s}\left(\mathbf{k}_{\|}, k_{\perp}\right)=\int_{-\infty}^{\infty} d \Delta z \tilde{V}\left(\mathbf{k}_{\|}, \Delta z\right) \theta(\Delta z) e^{i k_{\perp} \Delta z}=\hat{V}_{R}\left(\mathbf{k}_{\|}, k_{\perp}\right)+i \hat{V}_{I}\left(\mathbf{k}_{\|}, k_{\perp}\right) .
$$


In (14) and below, a three-dimensional Fourier transform is indicated by a hat, to distinguish it form the two-dimensional Fourier transform indicated by a tilde.

Let us first focus on the real part of $\hat{V}_{s}\left(\mathbf{k}_{\|}, k_{\perp}\right)$,

$$
\hat{V}_{R}\left(\mathbf{k}_{\|}, k_{\perp}\right)=\int_{0}^{\infty} d \Delta z \tilde{V}\left(\mathbf{k}_{\|}, \Delta z\right) \cos \left(k_{\perp} \Delta z\right)=\frac{1}{2} \int_{-\infty}^{\infty} d \Delta z \tilde{V}\left(\mathbf{k}_{\|}, \Delta z\right) e^{i k_{\perp} \Delta z},
$$

where we used the property $\tilde{V}\left(\mathbf{k}_{\|}, \Delta z\right)=\tilde{V}\left(\mathbf{k}_{\|},-\Delta z\right)$. From the above and Eq.(9), we obtain

$$
\hat{V}_{R}\left(\mathbf{k}_{\|}, k_{\perp}\right)=\frac{1}{2} \int d \mathbf{r} V(\mathbf{r}) e^{i \mathbf{r} \cdot \mathbf{k}}=\frac{1}{2} \hat{U}\left(k^{2}\right)
$$

where $\mathbf{r}=\left(\mathbf{r}_{\|}, z\right), \mathbf{k}=\left(\mathbf{k}_{\|}, k_{\perp}\right)$, and we introduced the function $\hat{U}$ of $k^{2}=k_{\|}^{2}+k_{\perp}^{2}$, based on the fact that the Fourier transform of the interaction potential is an even function of $k$. We Taylor expand $\hat{U}\left(k_{\|}^{2}+k_{\perp}^{2}\right)$ in terms of $k_{\perp}^{2}$,

$$
\hat{V}_{R}\left(\mathbf{k}_{\|}, k_{\perp}\right)=\frac{1}{2} \sum_{n=0}^{\infty} U_{2 n}\left(k_{\|}\right) k_{\perp}^{2 n}
$$

In the next step we Fourier-transform $\hat{V}_{R}\left(\mathbf{k}_{\|}, k_{\perp}\right)$ given in Eq. (17) back to the real space in the direction perpendicular to the surface $z=0$, introduce the operator $\beta \hat{U}\left(k_{\|}^{2}-\frac{\partial^{2}}{\partial z^{2}}\right)$ acting on $\Delta \tilde{\zeta}$ according to the equation

$$
\hat{U}\left(k_{\|}^{2}-\frac{\partial^{2}}{\partial z^{2}}\right) \Delta \tilde{\zeta}\left(\mathbf{k}_{\|}, z\right)=\sum_{n=0}^{\infty}(-1)^{n} U_{2 n}\left(k_{\|}\right) \frac{\partial^{2 n} \Delta \tilde{\zeta}\left(\mathbf{k}_{\|}, z\right)}{\partial z^{2 n}},
$$

and obtain the corresponding contribution to the internal energy

$$
\beta \Delta U_{R}=\frac{1}{2} \int \frac{d \mathbf{k}_{\|}}{(2 \pi)^{2}} \int_{0}^{\infty} d z \Delta \tilde{\zeta}\left(-\mathbf{k}_{\|}, z\right) \beta \hat{U}\left(k_{\|}^{2}-\frac{\partial^{2}}{\partial z^{2}}\right) \Delta \tilde{\zeta}\left(\mathbf{k}_{\|}, z\right)
$$

Let us now focus on the imaginary part $V_{I}$ that is an odd function of $k_{\perp}$,

$$
\hat{V}_{I}\left(\mathbf{k}_{\|}, k_{\perp}\right)=\int_{0}^{\infty} d \Delta z \tilde{V}\left(\mathbf{k}_{\|}, \Delta z\right) \sin \left(k_{\perp} \Delta z\right)=\sum_{n=0}^{\infty} I_{2 n+1}\left(k_{\|}\right) k_{\perp}^{2 n+1},
$$

where

$$
I_{2 n+1}\left(k_{\|}\right)=\int_{0}^{\infty} d \Delta z \tilde{V}\left(k_{\|}, \Delta z\right) \frac{(-1)^{n} \Delta z^{2 n+1}}{(2 n+1) !}
$$

We Fourier-transform $\hat{V}_{I}\left(\mathbf{k}_{\|}, k_{\perp}\right)$ given by (20) back to the real space in the direction perpendicular to the surface $z=0$, introduce the operator $\hat{V}_{I}\left(k_{\|}, i \frac{\partial}{\partial z}\right)$ by

$$
\hat{V}_{I}\left(k_{\|}, i \frac{\partial}{\partial z}\right) \Delta \tilde{\zeta}\left(\mathbf{k}_{\|}, z\right)=\sum_{n=0}^{\infty}(-1)^{n} I_{2 n+1}\left(k_{\|}\right) \frac{\partial^{2 n+1} \Delta \tilde{\zeta}\left(\mathbf{k}_{\|}, z\right)}{\partial z^{2 n+1}}
$$


and obtain the following expression for the corresponding contribution to the internal energy

$$
\beta U_{I}=\int \frac{d \mathbf{k}_{\|}}{(2 \pi)^{2}} \int_{0}^{\infty} d z \Delta \tilde{\zeta}\left(-\mathbf{k}_{\|}, z\right) \hat{V}_{I}\left(k_{\|}, i \frac{\partial}{\partial z}\right) \Delta \tilde{\zeta}\left(\mathbf{k}_{\|}, z\right) .
$$

As seen from (22), $\beta U_{I}$ is real as it should be. Because of the odd derivatives in Eq.(22), however, Eq.(23) reduces after integration by parts to a surface term of the form

$$
\beta U_{I}=-\frac{1}{2} \int \frac{d \mathbf{k}_{\|}}{(2 \pi)^{2}} \sum_{n=0}^{\infty}(-1)^{n} \beta I_{2 n+1}\left(k_{\|}\right) \sum_{m=0}^{2 n}(-1)^{m} \Delta \tilde{\zeta}^{(m)}\left(-\mathbf{k}_{\|}, 0\right) \Delta \tilde{\zeta}^{(2 n-m)}\left(\mathbf{k}_{\|}, 0\right),
$$

where $\Delta \tilde{\zeta}^{(m)}\left(\mathbf{k}_{\|}, 0\right)$ denotes the $m$-th derivative of $\Delta \tilde{\zeta}$ with respect to its second argument $z$ at $z=0$.

The above mathematical transformations lead to the expression for the excess grand potential

$$
\begin{aligned}
\Delta \beta \Omega[\zeta] & =\frac{1}{2} \int \frac{d \mathbf{k}_{\|}}{(2 \pi)^{2}} \int_{0}^{\infty} d z \Delta \tilde{\zeta}\left(-\mathbf{k}_{\|}, z\right) \beta \hat{U}\left(k_{\|}^{2}-\frac{\partial^{2}}{\partial z^{2}}\right) \Delta \tilde{\zeta}\left(\mathbf{k}_{\|}, z\right) \\
& +\int d \mathbf{r}_{\|} \int_{0}^{\infty} d z g\left(\zeta_{b}, \Delta \zeta\left(\mathbf{r}_{\|}, z\right)\right)+\beta U_{e x t}+\beta U_{I},
\end{aligned}
$$

where $\hat{U}$ is defined in (18), $U_{\text {ext }}$ is given in (12) or (13), $g$ is defined in (11), and $\beta U_{I}$ is given in (24). The above form is convenient for derivation of the Euler-Lagrange (EL) equations.

\section{B. Euler-Lagrange equations}

Minimization of $\Delta \beta \Omega[\zeta]$ given by (25) leads to the equilibrium volume fraction in our MF theory. We follow the standard procedure and require that the part of $\Delta \beta \Omega[\zeta+\delta \zeta]-\Delta \beta \Omega[\zeta]$ linear in $\delta \tilde{\zeta}\left(\mathbf{k}_{\|}, z\right)$ vanishes. The EL equation obtained in this way has the form

$$
\beta \hat{U}\left(k_{\|}^{2}-\frac{\partial^{2}}{\partial z^{2}}\right) \Delta \tilde{\zeta}\left(\mathbf{k}_{\|}, z\right)+\int d \mathbf{r}_{\|} e^{i \mathbf{k}_{\|} \cdot \mathbf{r}_{\|}} g^{(1)}\left(\zeta_{b}, \Delta \zeta\left(\mathbf{r}_{\|}, z\right)\right)+\beta \tilde{V}_{e x t}\left(\mathbf{k}_{\|}, z\right)=0,
$$

where we have introduced the notation $g^{(n)}\left(\zeta_{b}, \Delta \zeta\right)=\partial^{n} g\left(\zeta_{b}, \Delta \zeta\right) / \partial \Delta \zeta^{n}$, with the derivative taken at the indicated value of the second argument. We expand $g^{(1)}\left(\zeta_{b}, \Delta \zeta\right)$ about $\Delta \zeta=0$, take into account that $g^{(1)}\left(\zeta_{b}, 0\right)=0$ (see (11) ), and after truncating the expansion at the second-order term, obtain the linearized EL equation

$$
\beta \hat{U}\left(k_{\|}^{2}-\frac{\partial^{2}}{\partial z^{2}}\right) \Delta \tilde{\zeta}\left(\mathbf{k}_{\|}, z\right)+A_{2}\left(\zeta_{b}\right) \Delta \tilde{\zeta}\left(\mathbf{k}_{\|}, z\right)+\beta \tilde{V}_{e x t}\left(\mathbf{k}_{\|}, z\right)=0,
$$

where we have introduced $A_{n}\left(\zeta_{b}\right)=g^{(n)}\left(\zeta_{b}, 0\right)=d^{n} \beta f_{h}\left(\zeta_{b}\right) / d \zeta_{b}^{n}$ for $n \geq 2$ to simplify the notation. In order to be able to calculate $\Delta \tilde{\zeta}\left(\mathbf{k}_{\|}, z\right)$, it remains to determine the boundary conditions (BC). 


\section{Boundary conditions}

In derivation of (26) , we have performed integration by parts to get rid of the derivatives of $\delta \tilde{\zeta}$ that appear because of the presence of the differential operator in (25). However, in this way additional boundary terms that are proportional to $\delta \tilde{\zeta}\left(\mathbf{k}_{\|}, 0\right)$ and its derivatives are generated. In the case of the semiinfinite system, the boundary conditions follow from the requirement that the surface contribution to $\Delta \beta \Omega[\zeta+\delta \zeta]-\Delta \beta \Omega[\zeta]$,

$$
\begin{array}{r}
\delta \beta \Omega_{\text {surf }}=\sum_{n=1}^{\infty}(-1)^{n}\left[\beta I_{2 n-1}\left(\mathbf{k}_{\|}\right) \sum_{m=0}^{2 n-2}(-1)^{m} \Delta \tilde{\zeta}^{(2 n-m-2)}\left(\mathbf{k}_{\|}, 0\right) \delta \tilde{\zeta}^{(m)}\left(\mathbf{k}_{\|}, 0\right)\right. \\
\left.+\frac{1}{2} \beta U_{2 n}\left(\mathbf{k}_{\|}\right) \sum_{m=0}^{2 n-1}(-1)^{m} \Delta \tilde{\zeta}^{(2 n-m-1)}\left(\mathbf{k}_{\|}, 0\right) \delta \tilde{\zeta}^{(m)}\left(\mathbf{k}_{\|}, 0\right)\right]
\end{array}
$$

coming from $\beta U_{I}$ as well as from the integration by parts mentioned above, vanishes. The first two $\mathrm{BC}$ (the terms proportional to $\delta \tilde{\zeta}\left(\mathbf{k}_{\|}, 0\right)$ and $\delta \tilde{\zeta}^{\prime}\left(\mathbf{k}_{\|}, 0\right)$ ), are

$$
\sum_{n=1}^{\infty}(-1)^{n}\left[\frac{1}{2} \beta U_{2 n}\left(k_{\|}\right) \Delta \tilde{\zeta}^{(2 n-1)}\left(\mathbf{k}_{\|}, 0\right)+\beta I_{2 n-1}\left(k_{\|}\right) \Delta \tilde{\zeta}^{(2 n-2)}\left(\mathbf{k}_{\|}, 0\right)\right]=0
$$

and

$$
\sum_{n=1}^{\infty}(-1)^{n}\left[\beta I_{2 n+1}\left(k_{\|}\right) \Delta \tilde{\zeta}^{(2 n-1)}\left(\mathbf{k}_{\|}, 0\right)-\frac{1}{2} \beta U_{2 n}\left(k_{\|}\right) \Delta \tilde{\zeta}^{(2 n-2)}\left(\mathbf{k}_{\|}, 0\right)\right]=0
$$

If (13) instead of (121) is assumed for $U_{\text {ext }}$, then the last term in (26) or (27) should be removed, and $(2 \pi)^{2} \delta\left(\mathbf{k}_{\|}\right) \beta h$ should be added to the LHS of (29). Additional BC are $\lim _{z \rightarrow \infty} \Delta \tilde{\zeta}^{(m)}\left(k_{\|}, z\right)=0$.

\section{Transverse and lateral structure}

The local excess volume fraction in the real-space or in the Fourier representation can be written in the form

$$
\Delta \zeta\left(\mathbf{r}_{\|}, z\right)=\Delta \zeta_{0}(z)+\phi\left(\mathbf{r}_{\|}, z\right)
$$

or

$$
\Delta \tilde{\zeta}\left(\mathbf{k}_{\|}, z\right)=(2 \pi)^{2} \delta\left(\mathbf{k}_{\|}\right) \Delta \zeta_{0}(z)+\tilde{\phi}\left(\mathbf{k}_{\|}, z\right)
$$

By $\Delta \zeta_{0}(z)$ we denote the excess volume fraction at the distance $z$ from the surface, averaged over the $(x, y)$ plane. The functions $\phi\left(\mathbf{r}_{\|}, z\right)$ and $\tilde{\phi}\left(\mathbf{k}_{\|}, z\right)$ in turn are associated with the 
long-range lateral order, i.e. with periodic density oscillations in the plane parallel to the confining wall and separated by the distance $z$ from it. With the above definition, we have $\int d \mathbf{r}_{\|} \phi\left(\mathbf{r}_{\|}, z\right)=0$ and $\tilde{\phi}(\mathbf{0}, z)=0$. In a similar way we separate the external potential into the homogeneous and the oscillatory parts

$$
\tilde{V}_{e x t}\left(\mathbf{k}_{\|}, z\right)=(2 \pi)^{2} \delta\left(\mathbf{k}_{\|}\right) V_{\text {ext }}(z)+\tilde{V}_{\|}\left(\mathbf{k}_{\|}, z\right)
$$

with $\tilde{V}_{\|}(\mathbf{0}, z)=0$.

Inserting (32) in (26) and separating terms proportional to $\delta\left(\mathbf{k}_{\|}\right)$, gives us for $k_{\|}=0$

$$
\beta \hat{U}\left(-\frac{\partial^{2}}{\partial z^{2}}\right) \Delta \zeta_{0}(z)+\sum_{n=0}^{\infty} \frac{g^{(n+1)}\left(\zeta_{b}, \Delta \zeta_{0}(z)\right)}{n ! A_{u}} \int_{A_{u}} d \mathbf{r}_{\|} \phi^{n}\left(\mathbf{r}_{\|}, z\right)+\beta V_{\text {ext }}(z)=0,
$$

and for $k_{\|} \neq 0$

$$
\beta \hat{U}\left(\mathbf{k}_{\|}^{2}-\frac{\partial^{2}}{\partial z^{2}}\right) \tilde{\phi}\left(\mathbf{k}_{\|}, z\right)+\int d \mathbf{r}_{\|} e^{-i \mathbf{k}_{\|} \cdot \mathbf{r}_{\|}} g^{(1)}\left(\zeta_{b}, \Delta \zeta_{0}(z)+\phi\left(\mathbf{r}_{\|}, z\right)\right)+\beta \tilde{V}_{\|}\left(\mathbf{k}_{\|}, z\right)=0 .
$$

The integral in (34) is over the area of the unit cell of the periodic structure, $A_{u}$. As seen from (34) and (35), the near-surface long-range lateral order and the average density in the planes parallel to the surface are coupled.

Depending on a thermodynamic state, the long-range order near the confining wall, i.e. a structure periodic in the lateral direction, may or may not be present. We shall focus on the latter case, where only short-range lateral order is present near the surface. In fact for temperature and density considered in Ref. [44], only short-range lateral order at the surface was observed in MD simulations.

For $\phi=0$, Eq.(34) takes the simple form

$$
\beta \hat{U}\left(-\frac{\partial^{2}}{\partial z^{2}}\right) \Delta \zeta_{0}(z)+g^{(1)}\left(\zeta_{b}, \Delta \zeta_{0}(z)\right)+\beta V_{e x t}(z)=0 .
$$

Eqs(25)-(36) can be a starting point for various approximate theories, based on truncated expansions of $\beta \hat{U}, g^{(1)}$ and/or $\beta U_{I}$.

\section{E. Linearized EL equation in the absence of long-range lateral order}

$g^{(1)}\left(\zeta_{b}, \Delta \zeta_{0}(z)\right)$ in Eq.(36) can be Taylor expanded, and for small values of $\Delta \zeta_{0}(z)$, the expansion can be truncated at the first-order term. The linearized Eq.(36) then takes the simple form

$$
\beta \hat{U}\left(-\frac{\partial^{2}}{\partial z^{2}}\right) \Delta \zeta_{0}(z)+A_{2}\left(\zeta_{b}\right) \Delta \zeta_{0}(z)+\beta V_{e x t}(z)=0 .
$$


If $V_{\text {ext }}(z)=h \delta(z)$, the equation (37) becomes even simpler, and the solution is a sum of terms proportional to $\exp (i \alpha z)$, where $\alpha$ is a solution of the equation

$$
\beta \hat{U}\left(\alpha^{2}\right)+A_{2}\left(\zeta_{b}\right)=0 .
$$

In the disordered phase this equation has no solutions for real $\alpha$, because the disordered phase is stable when $\beta \hat{U}\left(k_{0}^{2}\right)+A_{2}\left(\zeta_{b}\right)>0$, where $\hat{U}$ takes a minimum at $k^{2}=k_{0}^{2}$. As the linearized equation can be valid for small $\Delta \zeta_{0}(z)$, i.e. for large $z$, the asymptotic decay is given by the solution $\alpha=i \alpha_{0} \pm \alpha_{1}$ with the imaginary part $\alpha_{0}>0$ with the smallest magnitude.

The correlation function in the bulk, $G(\Delta \mathbf{r})=\langle\zeta(\mathbf{r}) \zeta(\mathbf{r}+\Delta \mathbf{r})\rangle-\zeta_{b}^{2}$, is inversely proportional to the second functional derivative of the grand potential with respect to local deviations of the volume fraction from the average value. In Fourier representation $\hat{G}(k)$ is inversely proportional to the LHS of Eq.(38) with $\alpha=k$. Poles of $\hat{G}(k)$, i.e. zeros of the LHS of Eq.(38), determine the decay of correlations in the real space representation. This result shows that the decay length and the period of damped oscillations are the same in the correlation function in the bulk and in the density profile near a flat wall. This observation was confirmed by simulations of a particular version of the SALR model in Ref.[44].

There exist two possible cases: (i) $\alpha_{1}=0$ and $\Delta \zeta_{0}(z)$ decays monotonically, or (ii) $\alpha_{1} \neq 0$ and an oscillatory decay of $\Delta \zeta_{0}(z)$ takes place for large $z$. The first case concerns $\hat{U}\left(k^{2}\right)$ that takes the global minimum for $k=0$, and has the expansion $\hat{U}(0)+U_{2} k^{2}+\ldots$ with $\hat{U}(0)<0$,

$U_{2}>0$. The second case concerns $\hat{U}\left(k^{2}\right)$ that takes the global minimum for $k=k_{0}>0$, and has the expansion about the minimum $\hat{U}\left(k_{0}^{2}\right)+v\left(k^{2}-k_{0}^{2}\right)^{2}+\ldots$. In the first case, when the expansion of $\hat{U}\left(k^{2}\right)$ is truncated at the term proportional to $k^{2}$, our theory reduces to the standard Landau theory. In the second case, when $\hat{U}\left(k^{2}\right)$ is approximated by

$$
\hat{U}\left(k^{2}\right)=\hat{U}\left(k_{0}^{2}\right)+v\left(k^{2}-k_{0}^{2}\right)^{2}+\ldots,
$$

our theory reduces to the Landau-Brazovskii theory.

In the rest of the work we limit ourselves to the Brazovskii-type theory.

\section{F. Correlation function}

Let us focus on the correlation function in the case of no long-range lateral order $(\phi=0)$. In the mixed representation, $\tilde{G}\left(k_{\|} \mid z_{1}, z_{2}\right)$ is the correlation function betweem the volume- 
fraction waves with the wavelength $k_{\|}$in the longitudinal direction in the planes at the separations $z_{1}$ and $z_{2}$ from the wall. $\tilde{G}\left(k_{\|} \mid z_{1}, z_{2}\right)$ describes also the response in the plane at $z=z_{1}$ to an oscillatory perturbation with the wavelength $k_{\|}$in the plane at $z=z_{2}$. It is convenient to calculate this function from the relation

$$
\tilde{G}\left(\mathbf{k}_{\|} \mid z_{1}, z_{2}\right)=\frac{\delta \Delta \tilde{\zeta}\left(\mathbf{k}_{\|}, z_{1}\right)}{\delta\left(-\beta \tilde{V}_{\text {ext }}\left(\mathbf{k}_{\|}, z_{2}\right)\right)} .
$$

In order to obtain an equation for $\tilde{G}\left(\mathbf{k}_{\|} \mid z_{1}, z_{2}\right)$, we proceed as in the case of the Landau theory for simple fluids (see for example Ref.[49]), and perform functional differentiation of Eq.(26) with respect to $-\beta \tilde{V}_{\text {ext }}$. As a result we obtain the equation for the correlation function in the absence of the long-range lateral order and near a homogeneous wall

$$
\left[\beta \hat{U}\left(k_{\|}^{2}-\frac{\partial^{2}}{\partial z_{1}^{2}}\right)+g^{(2)}\left(\zeta_{b}, \Delta \zeta_{0}\left(z_{1}\right)\right)\right] \tilde{G}\left(k_{\|} \mid z_{1}, z_{2}\right)=\delta\left(z_{1}-z_{2}\right),
$$

where we took into account that the correlation function depends only on $k_{\|}=\left|\mathbf{k}_{\|}\right|$.

$\mathrm{BC}$ for $G$ can be obtained by functional differentiation of the BC for $\Delta \tilde{\zeta}\left(\mathbf{k}_{\|}, z\right)$, and from (29) we obtain

$$
\sum_{n=1}^{\infty}(-1)^{n}\left[\frac{1}{2} \beta U_{2 n}\left(k_{\|}\right) \tilde{G}^{(2 n-1)}\left(k_{\|} \mid 0, z_{2}\right)+\beta I_{2 n-1}\left(k_{\|}\right) \tilde{G}^{(2 n-2)}\left(k_{\|} \mid 0, z_{2}\right)\right]=0,
$$

where $\tilde{G}^{(m)}\left(k_{\|} \mid 0, z_{2}\right)$ denotes the $m$-th derivative with respect to $z_{1}$ at $z_{1}=0$. The BC for $z_{1} \rightarrow \infty$ is $\lim _{z_{1} \rightarrow \infty} \tilde{G}\left(k_{\|} \mid z_{1}, z_{2}\right)=0$.

Functional differentiation of the linearized equation for $G$ (Eq.(27)) with respect to $-\beta \tilde{V}_{\text {ext }}$, gives the equation for the correlation function in the Gaussian approximation,

$$
\left[\beta \hat{U}\left(k_{\|}^{2}-\frac{\partial^{2}}{\partial z_{1}^{2}}\right)+A_{2}\left(\zeta_{b}\right)\right] \tilde{G}\left(k_{\|} \mid z_{1}, z_{2}\right)=\delta\left(z_{1}-z_{2}\right),
$$

where $\hat{U}$ and $A_{n}$ are defined in Eq.(18) and below Eq.(27), respectively. Alternatively, Eq.(43) can be obtained from (25) and the analog of the Ornstein-Zernike equation

$$
\int d z^{\prime} \tilde{C}\left(\mathbf{k}_{\|}, z_{1}, z^{\prime}\right) \tilde{G}\left(k_{\|}, z^{\prime}, z_{2}\right)=\delta\left(z_{1}-z_{2}\right)
$$

where

$$
\tilde{C}\left(\mathbf{k}_{\|}, z_{1}, z^{\prime}\right)=\frac{\delta^{2} \Delta \beta \Omega}{\delta \Delta \tilde{\zeta}\left(-\mathbf{k}_{\|}, z_{1}\right) \delta \Delta \tilde{\zeta}\left(\mathbf{k}_{\|}, z^{\prime}\right)} .
$$

Note that Eq.(41) shows that the effect of the volume-fraction profile on the local structure is significant. However, this effect cannot be determined on the level of the Gaussian 
approximation (43). In this approximation, $\tilde{G}\left(k_{\|} \mid z_{1}, z_{2}\right)$ is independent of the excess volume fraction profile and hence on the wall-particle interactions. Thus, the solution of Eq.(43) cannot accurately describe the close vicinity of the wall. Particularly large inaccuracy of $\tilde{G}\left(k_{\|} \mid z_{1}, z_{2}\right)$ obtained from Eq.(43) is expected for large wall-particle interactions leading to large $\Delta \zeta_{0}$.

We assume small $\Delta \zeta_{0}$ in (41), i.e. small wall-fluid potential and/or large $z$, truncate the expansion of $g^{(2)}\left(\zeta_{b}, \Delta \zeta_{0}\left(z_{1}\right)\right)$, and obtain the equation

$$
\left[\beta \hat{U}\left(k_{\|}^{2}-\frac{\partial^{2}}{\partial z_{1}^{2}}\right)+A_{2}\left(\zeta_{b}\right)+A_{3}\left(\zeta_{b}\right) \Delta \zeta_{0}\left(z_{1}\right)+O\left(\Delta \zeta_{0}\left(z_{1}\right)^{2}\right)\right] \tilde{G}\left(k_{\|} \mid z_{1}, z_{2}\right)=\delta\left(z_{1}-z_{2}\right)
$$

If $A_{3}\left(\zeta_{b}\right) \Delta \zeta_{0}\left(z_{1}\right)$ is small, the term $A_{3}\left(\zeta_{b}\right) \Delta \zeta_{0}\left(z_{1}\right) \tilde{G}\left(k_{\|} \mid z_{1}, z_{2}\right)$ can be treated as a perturbation. $A_{3}\left(\zeta_{b}\right)$ takes large values for small and large $\zeta_{b}$, but vanishes for the critical volume fraction $\zeta_{b} \approx 0.129$. Thus, the approximation (43) is more accurate for $\zeta_{b} \approx 0.129$, where lamellar structure is expected at low $T$, than for very dilute systems, where clusters are formed. We will limit ourselves to the approximate equation (43) that gives the asymptotic decay of correlations at large distances $z$ from the wall, and can be easily solved analytically.

\section{THE GENERIC MODEL FOR INHOMOGENEOUS SYSTEMS}

Density waves with the wavenumber $k_{0}$ corresponding to the minimum of $\hat{V}(k)$ lead to the lowest internal energy. The waves associated with significantly larger energy occur with significantly smaller probability. We assume, following Ref. [4, 6] that the density waves with the wavenumbers significantly different from $k_{0}$ are much less probable. If such density waves can be disregarded, then $\hat{U}\left(k^{2}\right)$ can be approximated by (39). The operator $\hat{U}\left(k_{\|}^{2}-\frac{\partial^{2}}{\partial z^{2}}\right)$ defined in Eq.(18), in this lowest-order nontrivial approximation takes the form

$$
\hat{U}\left(k_{\|}^{2}-\frac{\partial^{2}}{\partial z^{2}}\right)=v \frac{\partial^{4}}{\partial z^{4}}-2 v\left(k_{\|}^{2}-k_{0}^{2}\right) \frac{\partial^{2}}{\partial z^{2}}+v\left(k_{\|}^{2}-k_{0}^{2}\right)^{2}+\hat{U}\left(k_{0}^{2}\right) .
$$

In the consistent approximation, we truncate the expansion of $\hat{V}_{I}\left(\mathbf{k}_{\|}, k_{\perp}\right)$ (see (20) ) at the lowest-order term, and obtain

$$
\hat{V}_{I}\left(\mathbf{k}_{\|}, k_{\perp}\right)=I_{1}\left(k_{\|}\right) k_{\perp}
$$

where $I_{1}\left(k_{\|}\right)=\int_{0}^{\infty} d z z \tilde{V}\left(k_{\|}, z\right)$ (see (21) $)$. Eqs.(47)-(48) define the generic model (GM) for a semiinfinite system with inhomogeneities at the length scale $2 \pi / k_{0}$. Note that apart from 
the external field term, there is only one surface term in this approximation (see (24)),

$$
\beta U_{I}=-\frac{\beta}{2} \int d \mathbf{k}_{\|} I_{1}\left(k_{\|}\right) \Delta \tilde{\zeta}\left(\mathbf{k}_{\|}, 0\right)^{2} .
$$

Moreover, the bulk interactions are characterized by just three numbers, $\hat{U}\left(k_{0}^{2}\right), k_{0}$ and $v$ (see (39)). As in the standard Landau theory, $\beta U_{I}$ describes the missing-neighbors contribution at the surface. There is an important difference between the simple fluids and the systems with the SALR interactions, however. Namely, $-I_{1}(0)>0$ for the attractive interactions, but in the SALR systems $-I_{1}(0)<0$ is expected when the repulsion is strong enough. In the first case the attraction by the particles in the bulk is not compensated by the particles missing for $z<0$, while in the second case the repulsion is not compensated. These unbalanced interactions lead to effective repulsion by the confining wall in simple fluids, and to effective attraction in the SALR systems. This effective attraction to a hard wall, due to the missing neighbors was indeed observed in simulations [35]. In the following, we present the EL equations and the solutions of these equations for the GM.

\section{A. The volume-fraction profile in the generic model}

When the external potential is localized at the surface, the EL equation for the excess density and the BC, Eqs.(36) and (29)-(30), simplify to

$$
\begin{gathered}
\beta\left(v \frac{\partial^{4}}{\partial z^{4}}+2 v k_{0}^{2} \frac{\partial^{2}}{\partial z^{2}}+v k_{0}^{4}+\hat{U}\left(k_{0}^{2}\right)\right) \Delta \zeta_{0}(z)+g^{(1)}\left(\zeta_{b}, \Delta \zeta_{0}(z)\right)=0, \\
{\left.\left[\frac{v}{2} \frac{\partial^{3}}{\partial z^{3}}+v k_{0}^{2} \frac{\partial}{\partial z}-I_{1}(0)\right] \Delta \zeta_{0}(z)\right|_{z=0}+h=0,}
\end{gathered}
$$

and

$$
\left.\left[\frac{v}{2} \frac{\partial^{2}}{\partial z^{2}}+2 k_{0}^{2}\right] \Delta \zeta_{0}(z)\right|_{z=0}=0 .
$$

The linearized equation (50) is simply

$$
\left(\frac{\partial^{4}}{\partial z^{4}}+2 k_{0}^{2} \frac{\partial^{2}}{\partial z^{2}}+D_{0}\right) \Delta \zeta_{0}(z)=0
$$

where

$$
D_{0}=\tau^{2}+k_{0}^{4}
$$


with

$$
\tau^{2}=\left(\hat{U}\left(k_{0}^{2}\right)+k_{B} T A_{2}\left(\zeta_{b}\right)\right) / v
$$

Here we limit ourselves to the linearized EL equation (53). The stability condition of the disordered phase, $\beta \hat{U}\left(k_{0}^{2}\right)+A_{2}\left(\zeta_{b}\right)>0$, implies that $D_{0}>0$. Since $D_{0}>0$ in the disordered phase, the solution of (53) in this phase is

$$
\Delta \zeta_{0}(z)=\mathcal{A} e^{-\alpha_{0} z} \cos \left(\alpha_{1} z+\vartheta\right)
$$

where

$$
\begin{aligned}
& \alpha_{0,1}=\left[\frac{1}{2}\left(\sqrt{D_{0}} \mp k_{0}^{2}\right)\right]^{1 / 2}, \\
& \mathcal{A}=-\frac{2 \beta h \sqrt{D_{0}}}{\alpha_{1}\left(v D_{0}-4 \alpha_{0} I_{1}(0)\right)},
\end{aligned}
$$

and

$$
\tan (\vartheta)=-\frac{k_{0}^{2}}{\tau}
$$

The oscillatory decay (56) at sufficiently large separations is quite universal for systems with inhomogeneities at the well-defined length scale (here $2 \pi / k_{0}$ ). The formula (56) fits quite well the simulation results for a particular version of the SALR interactions already for $z>2 \pi / \alpha_{1}$ [44]. The same behavior was predicted for the asymptotic decay of the charge density near a charged wall in ionic systems [50]. In this case, Eq.(56) fits very well simulation results already for $z>2 \pi / \alpha_{1}$, too [51]. The values of the parameters in (566), however, agree with simulations performed in Ref [44]. only semi-quantitatively. A better agreement with simulations was obtained when $\alpha$ was calculated form Eq.(38), and the effect

of clustering on the entropy was taken into account [44]. Quantitative agreement, however, cannot be expected, because (i) the gradient expansion restricts the results to long-ranged features, (ii) the presented theory is of MF type, and (iii) the entropic contribution is based on the reference system of hard spheres in the local density approximation.

\section{B. Correlation function in the generic model}

In the GM the EL equation and the BC for $\tilde{G}$ are (see Eqs.(41) and (42))

$$
\beta\left[v \frac{\partial^{4}}{\partial z_{1}^{4}}+2 v\left(k_{0}^{2}-k_{\|}^{2}\right) \frac{\partial^{2}}{\partial z_{1}^{2}}+d\left(z_{1}, k_{\|}\right)\right] \tilde{G}\left(k_{\|} \mid z_{1}, z_{2}\right)=\delta\left(z_{1}-z_{2}\right)
$$


where $d\left(z_{1}, k_{\|}\right)=\hat{U}\left(k_{0}^{2}\right)+v\left(k_{\|}^{2}-k_{0}^{2}\right)^{2}+k_{B} T g^{(2)}\left(\zeta_{b}, \Delta \zeta_{0}\left(z_{1}\right)\right)$ and

$$
\left.\left[\frac{v}{2} \frac{\partial^{3}}{\partial z^{3}}-v\left(k_{\|}^{2}-k_{0}^{2}\right) \frac{\partial}{\partial z}-I_{1}\left(k_{\|}\right)\right] \tilde{G}\left(k_{\|} \mid z, z^{\prime}\right)\right|_{z=0}=0 .
$$

In the Gaussian approximation, Eq.(60) simplifies to (see Eq.(43))

$$
\beta v\left[\frac{\partial^{4}}{\partial z_{1}^{4}}+2\left(k_{0}^{2}-k_{\|}^{2}\right) \frac{\partial^{2}}{\partial z_{1}^{2}}+D\left(k_{\|}^{2}\right)\right] \tilde{G}\left(k_{\|} \mid z_{1}, z_{2}\right)=\delta\left(z_{1}-z_{2}\right)
$$

where

$$
D\left(k_{\|}^{2}\right)=\tau^{2}+\left(k_{\|}^{2}-k_{0}^{2}\right)^{2},
$$

and $\tau$ is defined in (55). Because in the disordered phase $D\left(k_{\|}^{2}\right)>0$, the solution of (62) should have the form

$$
\begin{aligned}
\tilde{G}\left(k_{\|} \mid z_{1}, z_{2}\right) & =a_{-}\left(k_{\|}\right) e^{-\alpha\left(k_{\|}\right)\left|z_{1}-z_{2}\right|}+a_{-}^{*}\left(k_{\|}\right) e^{-\alpha^{*}\left(k_{\|}\right)\left|z_{1}-z_{2}\right|} \\
& +a_{+}\left(k_{\|}\right) e^{-\alpha\left(k_{\|}\right)\left(z_{1}+z_{2}\right)}+a_{+}^{*}\left(k_{\|}\right) e^{-\alpha^{*}\left(k_{\|}\right)\left(z_{1}+z_{2}\right)}
\end{aligned}
$$

where $\alpha\left(k_{\|}\right)=\alpha_{0}\left(k_{\|}\right)+i \alpha_{1}\left(k_{\|}\right)$, with

$$
\alpha_{0,1}\left(k_{\|}\right)=\left[\frac{1}{2}\left(\sqrt{D\left(k_{\|}^{2}\right)} \pm\left(k_{\|}^{2}-k_{0}^{2}\right)\right)\right]^{1 / 2} .
$$

The function (64) with (65) satisfies Eq.(62) provided that

$$
a_{-}\left(k_{\|}\right)=-\frac{k_{B} T}{2 v\left(\alpha^{2}\left(k_{\|}\right)-\alpha^{* 2}\left(k_{\|}\right)\right) \alpha\left(k_{\|}\right)} .
$$

The $\mathrm{BC}$ determines the amplitude $a_{+}$,

$$
a_{+}\left(k_{\|}\right)=\frac{v \sqrt{D\left(k_{\|}\right)} \alpha^{*}\left(k_{\|}\right)+2 I_{1}\left(k_{\|}\right)}{v \sqrt{D\left(k_{\|}\right)} \alpha^{*}\left(k_{\|}\right)-2 I_{1}(k \|)} a_{-}\left(k_{\|}\right) .
$$

$\tilde{G}\left(k_{\|} \mid z_{1}, z_{2}\right)$ exhibits an oscillatory decay as a function of $\left|z_{1}-z_{2}\right|$ and $z_{1}+z_{2}$ with the same characteristic lengths in both cases, and Eq.(64) can be written in the equivalent form

$$
\begin{aligned}
\tilde{G}\left(k_{\|} \mid z_{1}, z_{2}\right) & =\mathcal{A}_{-}\left(k_{\|}\right) e^{-\alpha_{0}\left(k_{\|}\right)\left|z_{1}-z_{2}\right|} \cos \left[\alpha_{1}\left(k_{\|}\right)\left|z_{1}-z_{2}\right|+\theta_{-}\left(k_{\|}\right)\right] \\
& +\mathcal{A}_{+}\left(k_{\|}\right) e^{-\alpha_{0}\left(k_{\|}\right)\left(z_{1}+z_{2}\right)} \cos \left[\alpha_{1}\left(k_{\|}\right)\left(z_{1}+z_{2}\right)+\theta_{+}\left(k_{\|}\right)\right] .
\end{aligned}
$$

The involved dependence of the parameters $\mathcal{A}_{-}, \mathcal{A}_{+}, \theta_{-}, \theta_{+}$on $k_{\|}$will not be given here. 
The inverse decay length $\alpha_{0}\left(k_{\|}\right)$is an increasing function of $k_{\|}$, and takes the smallest value $\alpha_{0}$ for $k_{\|}=0$. Thus, in the $z$ direction the correlations between the $k_{\|}=0$ modes decay most slowly, and in the same way as $\Delta \zeta_{0}(z)$. In contrast, the wavelength of oscillations in the $z$ direction, $2 \pi / \alpha_{1}\left(k_{\|}\right)$, increases with increasing $k_{\|}$. For $k_{\|}<k_{0}$, we have $\alpha_{0}\left(k_{\|}\right)<\alpha_{1}\left(k_{\|}\right)$, i.e. pronounced oscillations of the correlation function in the transverse direction, while $\alpha_{0}\left(k_{\|}\right)>\alpha_{1}\left(k_{\|}\right)$for $k_{\|}>k_{0}$ (strongly damped oscillations in the transverse direction).

The first term in (68) depends only on the separation between the two parallel planes, and is independent of the distance from the wall. The Fourier transform of this term in the $z$ direction gives the bulk correlation function in Fourier representation. For $z_{1}=z_{2} \gg 1$, i.e. in a single plane away from the wall, Eq.(68) takes a maximum for $k_{\|}^{2}=k_{0}^{2}-\tau / \sqrt{3}$.

For finite $z_{1}, z_{2}$ the second term in Eq.(68), describing the effect of the confining wall, becomes important. The dependence of the amplitude and the phase on $k_{\|}$is quite complex, and depends on $I_{1}\left(k_{\|}\right)$that in turn depends on the shape of the interaction potential. We shall discuss $\tilde{G}\left(k_{\|} \mid z_{1}, z_{2}\right)$ in more detail for a particular form of $V(r)$ in the next section.

\section{GENERIC MODEL RESULTS FOR DOUBLE-YUKAWA POTENTIAL}

As an example we consider the popular double-Yukawa potential,

$$
V(r)=-\frac{K_{1}}{r} e^{-\kappa_{1} r}+\frac{K_{2}}{r} e^{-\kappa_{2} r}
$$

For the reference-system free-energy density we choose the Percus-Yevick approximation

$$
\beta f_{h}(\zeta)=\rho \ln (\rho)-\rho+\rho\left[\frac{3 \zeta(2-\zeta)}{2(1-\zeta)^{2}}-\ln (1-\zeta)\right],
$$

where $\rho=6 \zeta / \pi$.

In order to calculate the excess volume fraction and the correlation function, we need to express $k_{0}, \hat{U}\left(k_{0}\right), v$ and $I_{1}\left(k_{\|}\right)$in terms of $K_{1}, K_{2}, \kappa_{1}$ and $\kappa_{2}$. For the chosen potential, we can easily obtain analytical expressions (see Appendix).

We choose the parameters $K_{1}=1, K_{2}=0.2, \kappa_{1}=1, \kappa_{2}=0.5$ as in earlier works focused on the bulk properties [4, 7]. $K_{1}$ sets the energy unit, and we introduce dimensionless temperature $T^{*}=k_{B} T / K_{1}$. For this potential, large clusters are formed, since $\pi / k_{0} \approx 5$. In this lowest-order approximation, the dependence on the thermodynamic state is only through the single parameter $\tau$, which in turn depends on $k_{B} T A_{2}\left(\zeta_{b}\right)$ (see (55)). In MF, 
the instability with respect to periodic ordering occurs below the $\lambda$-line given by $\tau=0$. Beyond MF, however, such an instability is not present, therefore we calculate the excess volume-fraction profile and the correlation function mainly for $\tau=0.23$ that is well above the $\lambda$-line.

As discussed in sec ПF, the effect of $\Delta \zeta_{0}$ on the correlation function, neglected in (62), should be smaller for $\zeta_{b} \approx 0.129$ than for different volume fractions. Our choice of $\tau$ for $\zeta_{b}=0.129$ corresponds to $T^{*} \approx 0.134$ (at the $\lambda$-line $T^{*} \approx 0.126$ for $\zeta_{b}=0.129$ ). For the chosen interactions and $\tau$, the inverse decay length in the GM, $\alpha_{0} \approx 0.184$, is very close to $\alpha_{0} \approx 0.187$ obtained from Eq.(38). The accuracy of $\alpha_{1}$ is not as good, $\alpha_{1} \approx 0.626$ and $\alpha_{1} \approx 0.576$ in the GM and in Eq.(38), respectively. When $\tau$ increases from $\tau=0, \alpha_{0}$ in the GM and given by Eq.(38) both increase, but $\alpha_{1}$ in the GM increases, whereas $\alpha_{1}$ obtained from Eq.(38) decreases. The GM becomes less accurate when the system becomes less inhomogeneous. Still, given all the approximations, the GM works quite well compared to the linearized exact EL equation in the phase space region corresponding to inhomogeneities at a well-defined length scale. The excess volume fraction in the GM is shown for $\tau=0.23$ in Fig 1.

In Fig,2 the inverse lengths characterizing the decay of the correlation between the longitudinal $k$-modes in the planes at the distance $z=z_{1}$ and $z=z_{2}$ from the confining wall are shown. Note that the longitudinal density waves with the wavelengths larger than $2 \pi / k_{0}$ excited in one plane decay much more slowly in the transverse direction than the short-wavelength longitudinal density oscillations. The short-wavelength longitudinal fluctuations in one plane practically do not propagate to different layers of particles. While long-wavelength density oscillations correspond merely to displacements, reorientation or reshaping of clusters or layers, the density waves with the wavelengths shorter than $\pi / k_{0}$ correspond merely to disintegration of the aggregates.

In order to describe the short-range order in the planes parallel to the wall, we present the structure factor for $z_{1}=z_{2}=z$, i.e within planes parallel to the wall. In Fig 3 we present $\tilde{G}\left(k_{\|} \mid z, z\right)$ for $z=0$ and $z=\infty$, far from and close to the $\lambda$-line. The shape of $\tilde{G}\left(k_{\|} \mid z, z\right)$ follows from the fact that each term in (68) has a maximum for a different value of $k_{\|}$. The maximum of the first term is much broader than the maximum of the second term in (68). Since the second term in (68) vanishes for $z \rightarrow \infty$, this means significantly larger correlation length near the wall than in the bulk. Both peaks are broader for larger $\tau$, indicating shorter 


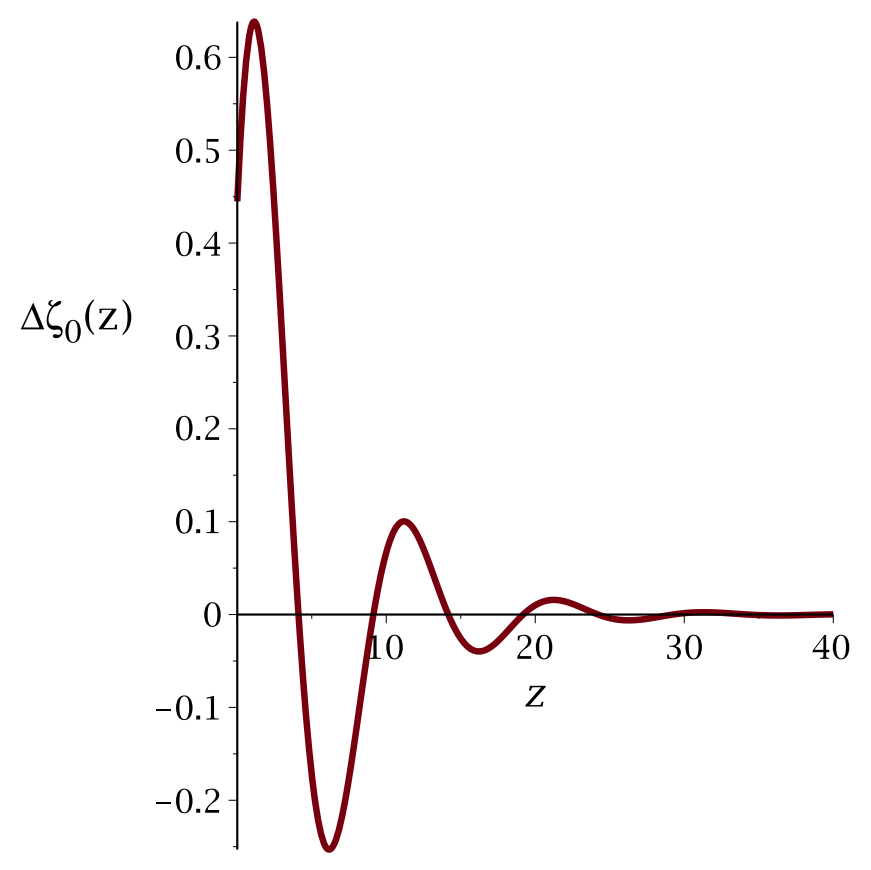

FIG. 1: The excess volume-fraction (56) for the PY reference system (70) and the interaction potential (69) with $K_{1}=1, K_{2}=0.2, \kappa_{1}=1, \kappa_{2}=0.5$. The thermodynamic state is given by $\tau=0.23$ with $\tau$ defined in Eq.(55). $\Delta \zeta_{0}$ is in units of the dimensionless wall-particle attraction, $\beta h$, and $z$ is in units of particle diameter.

correlation length away from the $\lambda$-line, both in the bulk and near the wall, as expected. The position of the maximum for $z \rightarrow \infty, k_{\|}=\left(k_{0}^{2}-\tau / \sqrt{3}\right)^{1 / 2}$, depends on $\tau$ much more strongly than the position of the maximum for $z=0$. For large $\tau$, the maxima of $\tilde{G}\left(k_{\|} \mid 0,0\right)$ and $\tilde{G}\left(k_{\|} \mid \infty, \infty\right)$ occur for similar $k_{\|}$. This means that in the case of weak inhomogeneities, the wavelength of the volume-fraction oscillations near the wall is similar to the wavelength of the oscillatory decay of correlations in the bulk. The stronger are the inhomogeneities in the bulk, i.e. the smaller is the value of $\tau$, the larger is the difference between the period of oscillations near the surface and in the bulk.

In Figs 4 and 5 we present $\tilde{G}\left(k_{\|} \mid z, z\right)$ for $\tau=0.23$, and for $z=1,2,4$ and $\infty$. For this intermediate value of $\tau$, the maximum for $z \rightarrow \infty$ is much broader and occurs at significantly larger value of $k_{\|}$than the maximum for $z \sim 1$. The range of the lateral periodic order is significantly larger near the wall, and the period of oscillations is larger too (Figs:6[7]).

As already discussed, we cannot expect accurate results for small $z$ in the Gaussian approximation that neglects the effect of the excess volume-fraction. The best accuracy is 


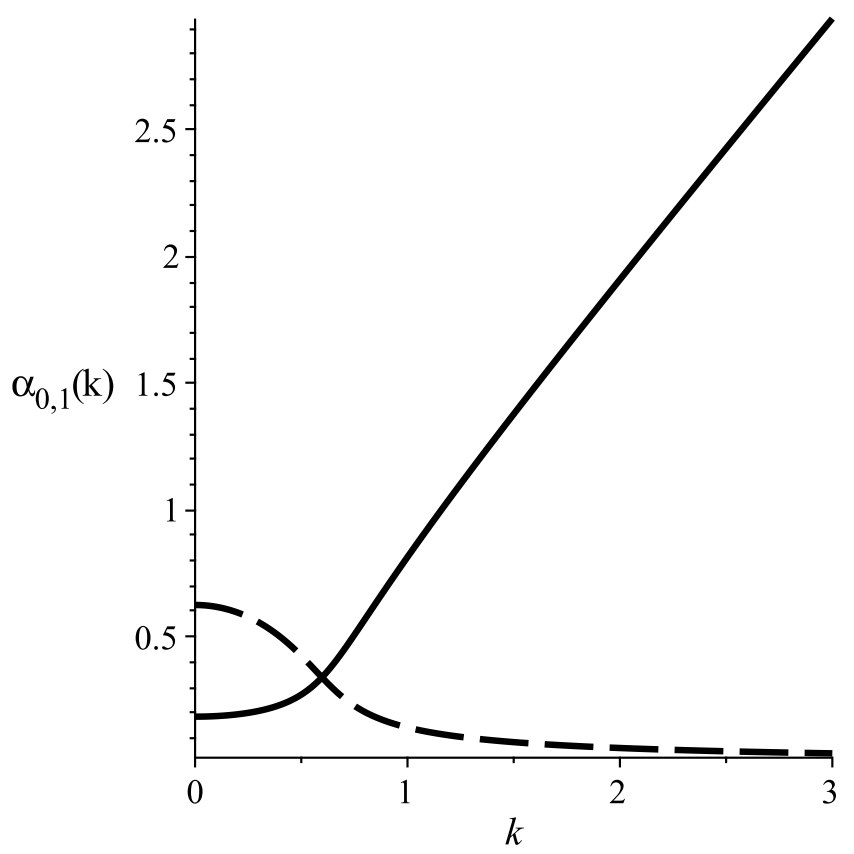

FIG. 2: The inverse decay length $\alpha_{0}(k)$ (solid line) and the wavenumber of the oscillatory decay $\alpha_{1}(k)$ (dashed line) in the direction perpendicular to the wall, of the correlations between the longitudinal $k$ modes (Eq.(65) and (66)). The interaction potential is given in Eq. (69) with $K_{1}=1, K_{2}=0.2, \kappa_{1}=1, \kappa_{2}=0.5$. The thermodynamic state is given by $\tau=0.23$ with $\tau$ defined in Eq.(55). For this potential, $k_{0} \approx 0.59$ and the wavelength of the most probable density-wave in the bulk is $2 \pi / k_{0} \approx 10$. Volume-fraction waves in the longitudinal direction with $k \gg 2 k_{0}$ correspond to disintegration of the aggregates. The decay length in the transverse direction of such fluctuations, $1 / \alpha_{0}(k)$, is smaller than the particle diameter, i.e. they do not propagate to another layer of particles.

expected for volume fractions corresponding to formation of layers (lamellar phase) at low $T^{*}$. Due to the missing-neighbors attraction to the wall, we may expect that the isotropic labyrinth of particle-rich region in the bulk becomes anisotropic near the wall, with a tendency of the layers of particles to assume the parallel orientation. Competition of this effect with the entropy leads to a larger wavelength of the oscillatory decay of density correlations in the longitudinal direction near the wall than in the bulk. 

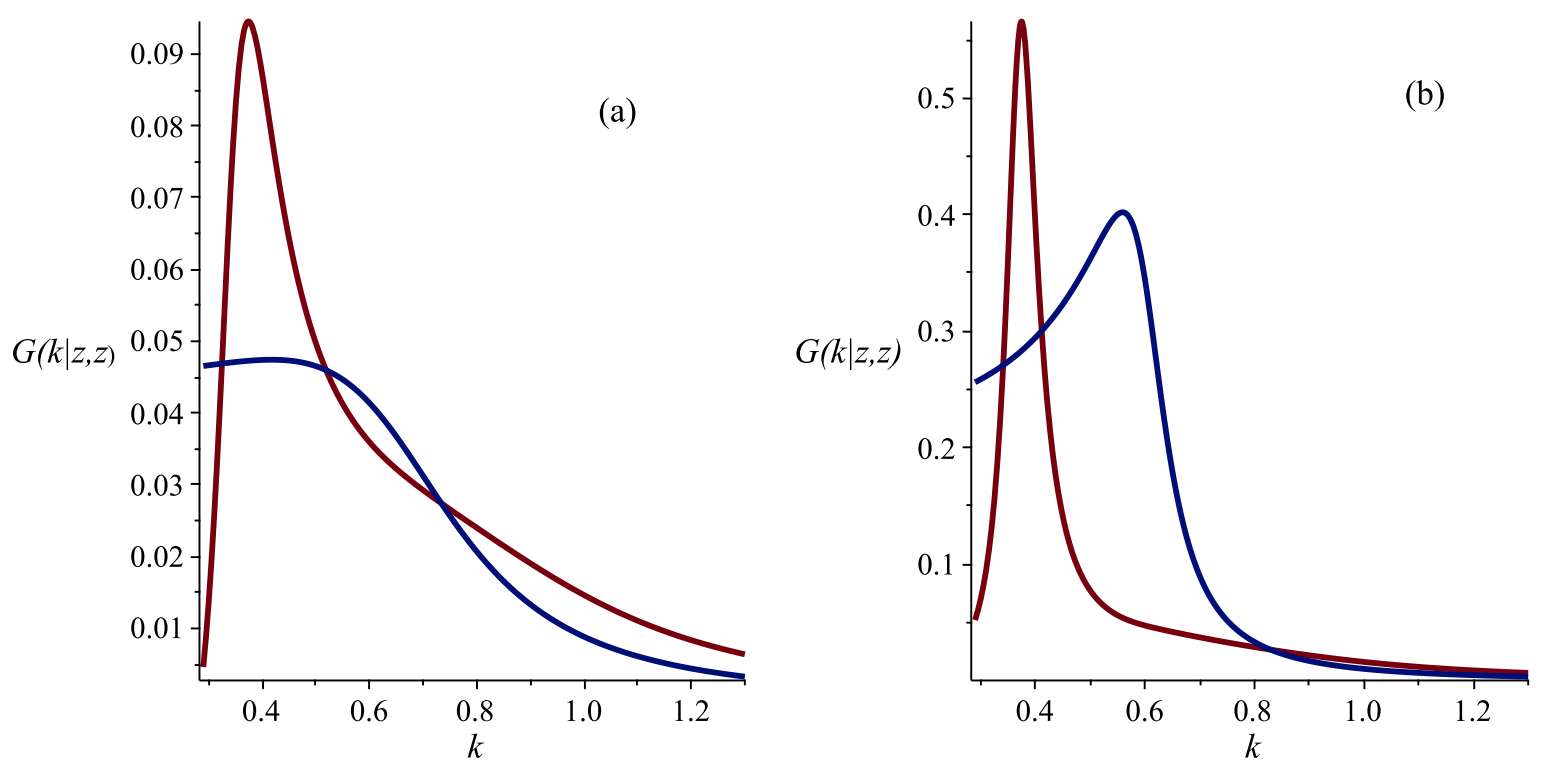

FIG. 3: The correlation function (68) in 2D Fourier representation in the planes parallel to the wall at $z=0$ (upper line), and $z=\infty$ (lower line) for the PY reference system and the interaction potential (69) with $K_{1}=1, K_{2}=0.2, \kappa_{1}=1, \kappa_{2}=0.5$. The thermodynamic state is given by $\tau=0.316$ (a) and $\tau=0.076$ (b) with $\tau$ defined in Eq. (55). The wavenumber is in units of inverse particle diameter.

\section{SUMMARY AND DISCUSSION}

We have developed a mesoscopic theory for self-assembling systems near a confining surface. We focused on the effects of the wall on a disordered inhomogeneous phase and limited ourselves to the MF approximation. In the first step, the standard DFT expression for the excess grand potential has been transformed to an equivalent form (Eq.(25) ) that consists of the bulk and the surface contributions. The surface contribution representing the missing neighbors beyond the confining surface (Eq.(24)), is expressed in terms of moments of the interaction potential (Eq.(21) ). Eq.(25) allowed for a derivation of the EL equations for the volume-fraction profile and the correlation function in the near-surface region (Eq.(26) and (41)), with the BC following from Eq.(28). Solutions of these equations should be the same as the results of minimization of the postulated excess grand potential.

In principle, ordered periodic structure in the lateral direction could be induced in the vicinity of the confining surface, and we obtained equations for the excess volume fraction at the distance $z$ from the surface, $\Delta \zeta_{0}(z)$, and for the modulations of the volume fraction in 


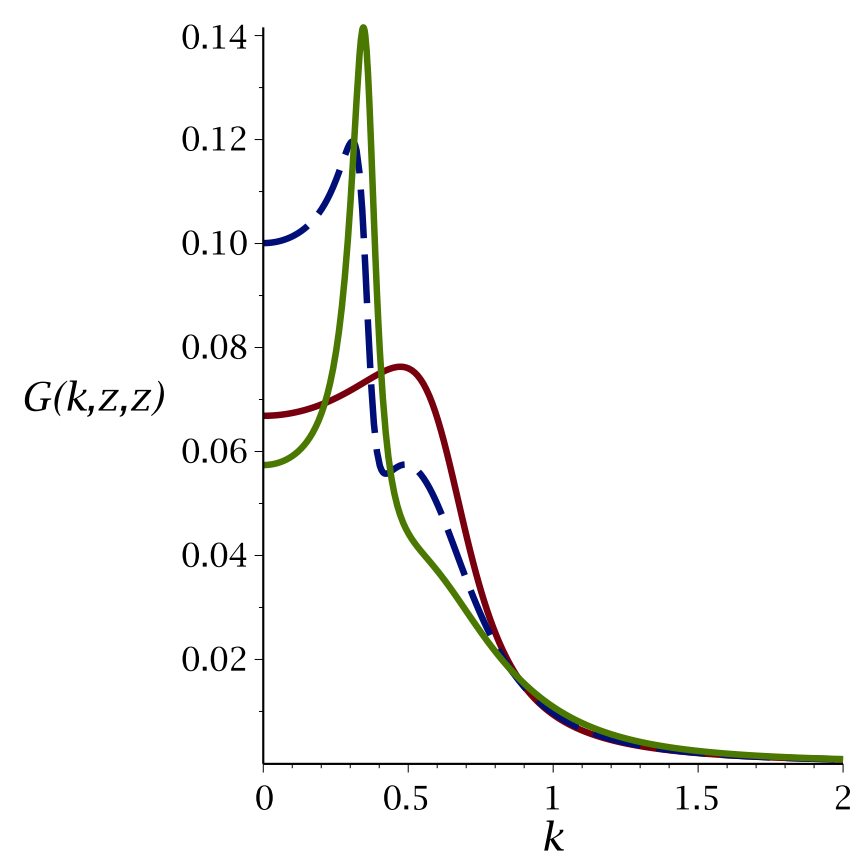

FIG. 4: The correlation function (68) in 2D Fourier representation in the planes parallel to the wall at $z=1$ (upper solid line), $z=2$ (dashed line) and $z=\infty$ (lower solid line) for the PY reference system and the interaction potential (69) with $K_{1}=1, K_{2}=0.2, \kappa_{1}=1, \kappa_{2}=0.5$. The thermodynamic state is given by $\tau=0.23$ with $\tau$ defined in Eq.(55). The wavenumber is in units of inverse particle diameter.

the planes parallel to the wall. In the rest of the paper we limited ourselves to the absence of the long-range order, however. In simulations, only short-range periodic structure was found for the considered thermodynamic states [44].

We next considered various approximate versions of the theory, especially the linearized equation for $\Delta \zeta_{0}(z)$ (Eq.(37)) that can be solved analytically. The analytical solution gives the asymptotic decay of the excess volume-fraction at large distances.

The lowest-order nontrivial approximation, GM, has been introduced in sec III. It is based on the same approximation for the interaction potential in Fourier representation (Eq.(39)) as in the theory for bulk systems with mesoscopic inhomogeneities [4, 48]. In addition, the series representing the missing-neighbors contribution to the excess grand potential associated with the presence of the confining surface (Eq.(24) $)$ is truncated at the first order term. In this approximation, the missing neighbors contribution to the grand potential is proportional to $\Delta \zeta_{0}(0)^{2}$. If in addition the wall-particle interaction is of very short range 


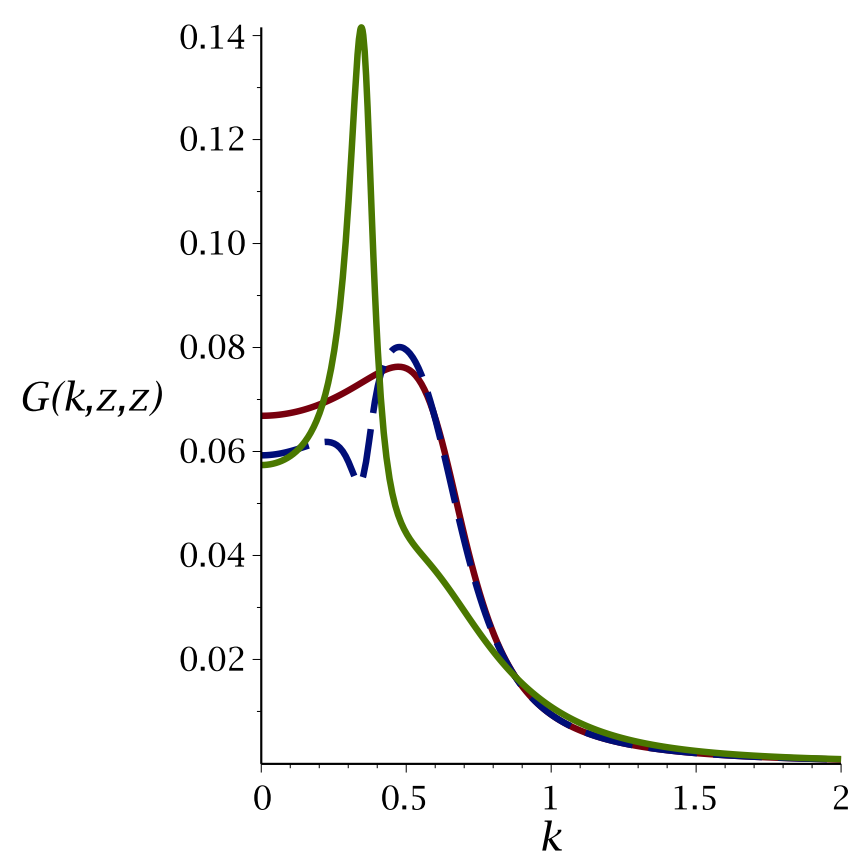

FIG. 5: The correlation function (68) in 2D Fourier representation in the planes parallel to the wall at $z=1$ (upper solid line), $z=4$ (dashed line) and $z=\infty$ (lower solid line) for the PY reference system and the interaction potential (69) with $K_{1}=1, K_{2}=0.2, \kappa_{1}=1, \kappa_{2}=0.5$. The thermodynamic state is given by $\tau=0.23$ with $\tau$ defined in Eq.(55).

and we can assume a contact potential, the mathematical form of the GM resembles strongly Landau-type theory for a semiinfinite system, with the bulk part of the Brazovskii form. However, in our theory there are no free phenomenological parameters. All coefficients depend on the interaction potential and on the thermodynamic state.

Solutions of the linearized equations in GM are presented and discussed on a general level

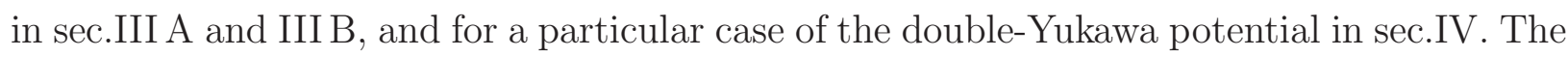
volume fraction profile has a form of exponentially damped oscillations, that very well reproduces results of simulations except from $z<2 \pi / \alpha_{1}$. The decay length and the wavenumber agree with simulation on a semiquantitative level. The GM quite well reproduces the solution of the more general equation (38) for the decay length. However, the wavenumber of oscillations deviates from the solution of (38) in the case of weak inhomogeneities (high $T^{*}$ ). The stronger the inhomogeneities, the better the agreement between GM and Eq.(37).

We have solved the equation for the correlation function only in GM and only in the Gaussian approximation. The correlation between volume fraction waves with the wave- 


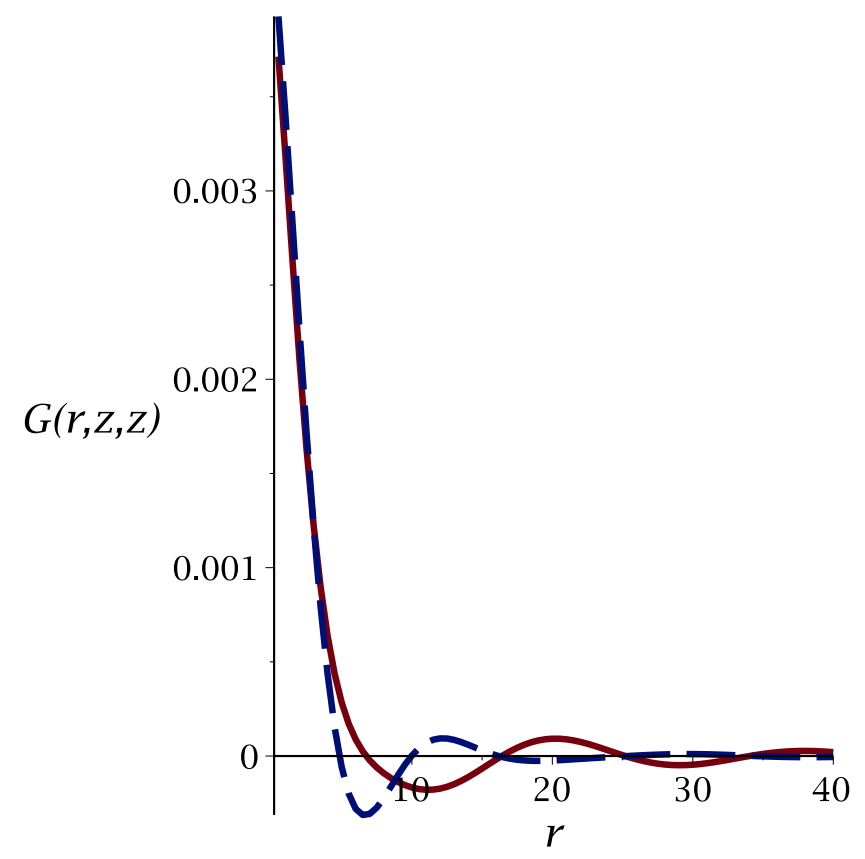

FIG. 6: The correlation function in real-space representation in the planes parallel to the wall at $z=1$ ( solid line), and $z=4$ (dashed line) for the PY reference system and the interaction potential (69) with $K_{1}=1, K_{2}=0.2, \kappa_{1}=1, \kappa_{2}=0.5$. The thermodynamic state is given by $\tau=0.23$ with $\tau$ defined in Eq.(55).

length $k_{\|}$in the planes at the distance $z_{1}$ and $z_{2}$ from the wall (Eq.(68)) consists of two terms. The first one is a function of $\left|z_{1}-z_{2}\right|$ and is independent of the surface properties. This is a kind of "background" bulk correlations, present for any distance from the wall. The second term is a function of $z_{1}+z_{2}$, and depends on the missing-neighbors contribution. This term is significant only close to the wall. Since the missing neighbors contribution depends on $\int_{0}^{\infty} d z z \tilde{V}\left(k_{\|}, z\right)$, the effect of the wall on the correlations depends on the shape of the interaction potential. Both terms in Eq.(68) exhibit oscillatory decay with the same characteristic lengths that strongly depend on $k_{\|}$. The volume fraction fluctuations in longitudinal direction with the wavelength shorter than the size of aggregates, $\sim \pi / k_{0}$, practically do not propagate to different layers. The largest range in the transverse direction (the same as the decay length of $\left.\Delta \zeta_{0}(z)\right)$ have the volume fraction fluctuations in the longitudinal direction with $k_{\|}=0$.

The short-range order in the planes parallel to the wall, described by $\tilde{G}\left(k_{\|} \mid z, z\right)$, has been investigated for the double-Yukawa potential, where we could obtain analytical results in 


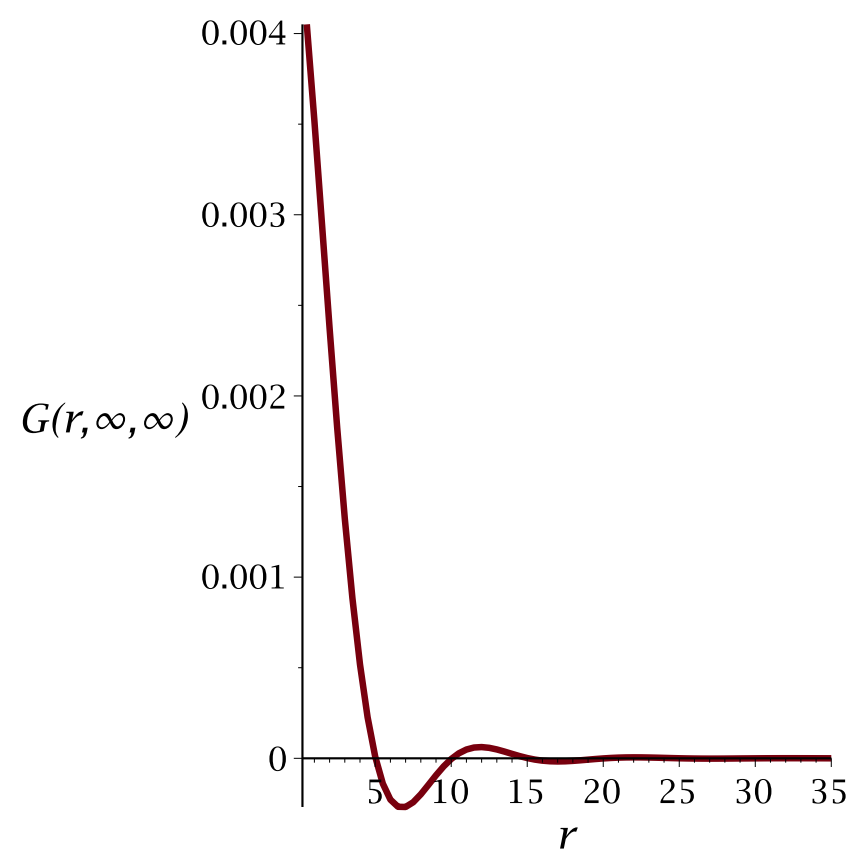

FIG. 7: The correlation function in real-space representation in the planes parallel to the wall at $z \rightarrow \infty$ for the PY reference system and the interaction potential (69) with $K_{1}=1, K_{2}=0.2, \kappa_{1}=$ $1, \kappa_{2}=0.5$. The thermodynamic state is given by $\tau=0.23$ with $\tau$ defined in Eq.(55).

the Gaussian approximation. Each term in $\tilde{G}\left(k_{\|} \mid z, z\right)$ has a maximum for a different value of $k_{\|}$. The maximum of the bulk term is much broader, indicting shorter decay length. Larger decay length in the longitudinal direction near the surface than far from it was observed in simulations [44, 45] in agreement with our predictions.

We presented analytical results for the simplest version of the theory. The analytical expressions allow to investigate asymptotic behavior and to draw general conclusions. We hope that the solutions discussed above show the key properties of the near-surface structure of the disordered phase in self-assembling systems. Thanks to the systematic derivation of various approximate versions of the theory, it is possible to obtain more accurate results for various model systems. The theory developed in this work can be a convenient tool for studies of the ordering effects of external surfaces on systems with spontaneous inhomogeneities on the mesoscopic length scale. First of all, it will be interesting to solve the nonlinear equations in GM. It is also of interest to extend the theory beyond MF, by taking into account the fluctuation contribution to the excess grand potential. To do so we shall generalize the theory developed in Ref.[48] to the semiinfinite system along the lines described in this work. 


\section{APPENDIX. THE PARAMETERS $k_{0}, \hat{U}\left(k_{0}^{2}\right), v$ AND $I_{1}\left(k_{\|}\right)$FOR THE DOUBLE- YUKAWA POTENTIAL}

In Fourier representation the potential (69) takes the form

$$
\hat{U}\left(k^{2}\right)=4 \pi\left[\frac{K_{2}}{\kappa_{2}^{2}+k^{2}}-\frac{K_{1}}{\kappa_{1}^{2}+k^{2}}\right] .
$$

The parameters in the Landau-Brazovskii type theory with $\hat{U}$ approximated by Eq. (39) are

$$
\begin{gathered}
k_{0}^{2}=\frac{\kappa_{1}^{2} \sqrt{K_{2}}-\kappa_{2}^{2} \sqrt{K_{1}}}{\sqrt{K_{1}}-\sqrt{K_{2}}}, \\
\hat{U}\left(k_{0}^{2}\right)=-4 \pi \frac{\left(\sqrt{K_{1}}-\sqrt{K_{2}}\right)^{2}}{\kappa_{1}^{2}-\kappa_{2}^{2}}, \\
v=4 \pi \frac{\left(\sqrt{K_{1}}-\sqrt{K_{2}}\right)^{4}}{\left(\kappa_{1}^{2}-\kappa_{2}^{2}\right)^{3} \sqrt{K_{1} K_{2}}} .
\end{gathered}
$$

The missing-neighbors interaction term (see (21) $)$ can be easily calculated analytically, and the result is

$$
I_{1}\left(k_{\|}\right)=2 \pi\left[\frac{K_{2}}{\left(\kappa_{2}^{2}+k_{\|}^{2}\right)^{3 / 2}}-\frac{K_{1}}{\left(\kappa_{1}^{2}+k_{\|}^{2}\right)^{3 / 2}}\right] .
$$

\section{ACKNOWLEDGEMENTS}

I would like to thank Vyacheslav Vikhernko for discussions, and Guillermo Zarragoicoechea, Ariel Meyra and Andres de Virgilis for discussions and hospitality at the Instituto de Fisica de Liquidos y Sistemas Biologicos, UNLP in La Plata, where a part of this work was done. This project has received funding from the European Union Horizon 2020 research and innovation programme under the Marie Skłodowska-Curie grant agreement No 734276 (CONIN). An additional support in the years 2017-2020 has been granted for the CONIN project by the Polish Ministry of Science and Higher Education. Financial support from the National Science Center under grant No. 2015/19/B/ST3/03122 is also acknowledged.

[1] M. Seul and D. Andelman, Science 267, 476 (1995). 
[2] M. W. Matsen and F. S. Bates, Macromolecules 29, 1091 (1996).

[3] A. Stradner et al., Nature 432, 492 (2004).

[4] A. Ciach, J. Pȩkalski, and W. T. Góźdź, Soft Matter 9, 6301 (2013).

[5] A. de Candia et al., Phys. Rev. E 74, 010403(R) (2006).

[6] A. Ciach, Phys. Rev. E 78, 061505 (2008).

[7] A. Ciach and W. T. Góźdź, Condens. Matter Phys. 13, 23603 (2010).

[8] D. Pini and A. Parola, Soft Matter 13, 9259 (2017).

[9] M. Edelmann and R. Roth, Phys. Rev. E 93, 062146 (2016).

[10] Y. Zhuang, K. Zhang, and P. Charbonneau, Phys. Rev. Lett. 116, 098301 (2016).

[11] Y. Zhuang and P. Charbonneau, J. Phys. Chem. B 120, 6178 (2016).

[12] A. Imperio and L. Reatto, J. Phys.: Condens. Matter 18, S2319 (2004).

[13] B. A. Lindquist, R. B. Jadrich, and T. M. Truskett, Soft Matter 12, 2663 (2016).

[14] M. B. Sweatman, R. Fartaria, and L. Lue, J. Chem. Phys. 140, (2014).

[15] A. J. Archer and N. B. Wilding, Phys. Rev. E 76, 031501 (2007).

[16] M. W. Matsen and M. Schick, Curr. Op. Colloid and Interf. Sci. 1, 329 (1996).

[17] T. H. Zhang, J. Groenewold, and W. K. Kegel, Phys. Chem. Chem. Phys. 11, 10827 (2009).

[18] A. I. Campbell, V. J.Anderson, J. S. van Duijneveldt, and P. Bartlett, Phys. Rev. Lett. 94, 208301 (2005).

[19] C. P. Royall, Soft Matter 14, 4020 (2018).

[20] M. Bergman, T. Garting, P. Schurtenberger, and A. Stradner, J. Phys. Chem. B 123, 2432 (2019).

[21] P. Pieranski, P. Sotta, D. Rohe, and M. Imperor-Clerc, Phys. Rev. Lett. 84, 2409 (2000).

[22] P. Pieranski, L. Sittler, P. Sotta, and M. Imperor-Clerc, 5, 317 (2001).

[23] L. Latypova, W. Gozdz, and P. Pieranski, The European Physical Journal E 36, 88 (2013)

[24] W. Gozdz, in Advances in Biomembranes and Lipid Self-Assembly, Vol. 23, edited by A. Iglic, C. V. Kulkarni, and M. Rappolt (Academic Press, 2016), pp. 145 - 168.

[25] P. Pieranski, Advances in Planar Lipid Bilayers and Liposomes 14, 1 (2011).

[26] M. W. Matsen, J. Chem. Phys. 106, 7781 (1997).

[27] M.Tasinkevych and A.Ciach, J. Chem. Phys. 115, 8705 (2001).

[28] A. J. Archer, Phys. Rev. E 78, 031402 (2008).

[29] L. Latypova, W. T. Gozdz, and P. Pieranski, Langmuir 30, 488 (2014), pMID: 24372147. 
[30] Y. Hu and P. Charbonneau, Soft Matter 14, 4101 (2018).

[31] M. Tasinkevych and A. Ciach, Phys. Rev. E 72, 061704 (2005).

[32] A. Ciach and M. Tasinkevych, Mol. Phys 109, 1101 (2011).

[33] A. Imperio and L. Reatto, Phys. Rev. E 76, 040402 (2007).

[34] N. G. Almarza, J. Pȩkalski, and A. Ciach, Soft Matter 12, 7551 (2016).

[35] H. Serna, W. T. Gozdz, and E. G. Noya, Langmuir 35, 702 (2019).

[36] J. Pȩkalski, E. Bildanau, and A. Ciach, Soft Matter 15, 7715 (2019).

[37] D. Antelmi, P. Kékicheff, and P. Richetti, J. Phys. II France 5, 103 (1995).

[38] D. A. Antelmi and P. Kékicheff, J. Phys. Chem. B 101, 8169 (1997).

[39] S. J. Richardson et al., Langmuir 30, 13510 (2014), pMID: 25346159.

[40] A.-C. Shi and B. Li, Soft Matter 9, 13981413 (2013).

[41] G. S. Doerk and K. G. Yager, Mol. Syst. Des. Eng. 2, 518538 (2017).

[42] W. T. Gozdz, Langmuir 31, 13321 (2015)

[43] A. Ciach, M. Tasinkevych, and A. Maciołek, Europhys Lett 45, 495 (1999).

[44] M. Litniewski and A. Ciach, J. Chem. Phys. 150, 234702 (2019).

[45] V. V. E. Bildanau, J. Pȩkalski and A. Ciach, arxiv.org/abs/1909.09374 (2019), preprint.

[46] A. J. Archer and A. Malijevsky, J. Phys.: Condens. Matter 28, 244017 (2016).

[47] A. P. Santos, J. Pȩkalski, and A. Z. Panagiotopoulos, Soft Matter 13, 8055 (2017).

[48] A. Ciach, Soft Matter 14, 5497 (2018).

[49] H. W. Diehl and A. Ciach, Phys. Rev. B 44, 6642 (1991).

[50] A. Ciach, J.Mol. Liquids 270, 138 (2018).

[51] J. M. Otero-Mato et al., Phys. Chem. Chem. Phys. 20, 30412 (2018). 